\title{
Computing stationary distributions in equilibrium and nonequilibrium systems with forward flux sampling
}

\author{
Chantal Valeriani ${ }^{\text {a) }}$ \\ FOM Institute for Atomic and Molecular Physics, Kruislaan 407, 1098 SJ Amsterdam, The Netherlands \\ Rosalind J. Allen \\ FOM Institute for Atomic and Molecular Physics, Kruislaan 407, 1098 SJ Amsterdam, The Netherlands \\ and SUPA, School of Physics, The University of Edinburgh, Mayfield Road, Edinburgh EH9 SJZ, \\ United Kingdom \\ Marco J. Morelli, Daan Frenkel, and Pieter Rein ten Wolde \\ FOM Institute for Atomic and Molecular Physics, Kruislaan 407, 1098 SJ Amsterdam, The Netherlands
}

(Received 25 May 2007; accepted 9 July 2007; published online 21 September 2007)

\begin{abstract}
We present a method for computing stationary distributions for activated processes in equilibrium and nonequilibrium systems using forward flux sampling. In this method, the stationary distributions are obtained directly from the rate constant calculations for the forward and backward reactions; there is no need to perform separate calculations for the stationary distribution and the rate constant. We apply the method to the nonequilibrium rare event problem proposed by Maier and Stein, to nucleation in a 2-dimensional Ising system, and to the flipping of a genetic switch. (C) 2007 American Institute of Physics. [DOI: 10.1063/1.2767625]
\end{abstract}

\section{INTRODUCTION}

Rare events are ubiquitous in physics, chemistry, and biology; examples include crystal nucleation, chemical reactions, and protein folding. Rare events are activated processes, for which the average waiting time between events can be orders of magnitude longer than the duration of the event itself. This makes these events intrinsically difficult to investigate experimentally. Computer simulations are therefore a natural tool to use-yet conventional numerical techniques are impractical for rare events, because most of the CPU time is wasted on the uneventful waiting time between events. A number of "rare event" simulation schemes have recently been developed in the field of soft-condensed matter physics, which makes it possible to zoom in on the rare events themselves. Techniques such as umbrella sampling allow the calculation of free-energy barriers separating the stable states, ${ }^{1-4}$ while schemes such the Bennet-Chandler method $^{5,6}$ also allow the computation of rate constants. Transition path sampling ${ }^{7-10}$ allows both rate constants and transition paths to be obtained. These techniques have been used for a wide range of applications including ion permeation through membranes, protein folding, and nucleation. However, these schemes require prior knowledge of the phasespace density. For systems that are in thermodynamic equilibrium -with detailed balance and microscopic reversibility - the phase-space density is known: it is given by the Boltzmann distribution. In contrast, for systems that are out of equilibrium, the phase-space density is usually not known. This means that most numerical techniques for simulating rare events are limited to equilibrium systems, and thus exclude a host of important rare-event problems in non-

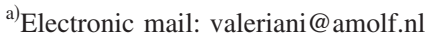

equilibrium systems, such as polymer collapse under flow, crystal nucleation under shear, and rare events in biology, such as protein translocation and switching events in biochemical networks. We have recently developed a numerical technique, called forward flux sampling (FFS) ${ }^{11-13}$ that makes it possible to compute rate constants in both equilibrium and nonequilibrium systems with stochastic dynamics. In this paper, we show how stationary distributions can also be obtained directly from a FFS calculation, for both equilibrium and nonequilibrium systems. For equilibrium systems the advantage is that from a FFS simulation one can obtain not only the rate constant, but also information about the free-energy landscape. For nonequilibrium systems the concept of free energy does not apply, but one can obtain the steady-state probability distribution as a function of a chosen order parameter (or order parameters). To our knowledge, this is the first method to be proposed for computing stationary distributions for multidimensional nonequilibrium systems that are in steady state.

In soft-condensed matter physics, the rate $k$ of an activated process in an equilibrium system is often written as the product of two factors,

$$
k=R\left(q^{*}\right) \rho\left(q^{*}\right) .
$$

Here, $q$ is an order parameter that connects the initial and final states, assuming that the system evolves between two states. It is defined such that for $q<q^{*}$, the system is in the initial state, while for $q>q^{*}$ it is in the final state. The quantity $\rho\left(q^{*}\right)$ is the probability that the system is at the dividing surface $q=q^{*}$ and $R\left(q^{*}\right)$ is the rate at which this dividing surface is crossed. For equilibrium systems, $\rho\left(q^{*}\right)$ is proportional to $\exp \left(-\beta \Delta G\left(q^{*}\right)\right)$, where $\beta$ is the inverse temperature and $\Delta G(q)$ is the (Landau) free-energy of the system as a function of the order parameter $q$. It is natural to locate the 
dividing surface $q^{*}$ at the top of the free-energy barrier $\Delta G(q)$ separating the two states. The rate constant is thus given by the probability of being at the top of the free-energy barrier, multiplied by a kinetic prefactor.

The Bennett-Chandler method for computing rate constants for activated processes uses a two-step procedure: ${ }^{5,6}$ one first computes the free-energy barrier, using, for example, the umbrella sampling scheme, ${ }^{1-4}$ and then the kinetic prefactor, using a molecular dynamics simulation in which trajectories are fired from the top of the free-energy barrier. However, this method is computationally demanding, and its success depends strongly on the choice of the reaction coordinate $q$. If $q$ is poorly chosen, the system will sample the wrong part of the phase space, which will not only conceal the mechanism of the transition, but also impede the computation of the rate constant; while the choice of $q$ coordinate does not affect the value of the rate constant $k$, it can strongly affect the efficiency with which $k$ is computed. For highdimensional complex systems it can be difficult to make a good choice for $q$, since this requires a priori insight into the reaction mechanism.

Transition-path sampling (TPS) has been developed to alleviate these problems. ${ }^{7,8,14,15}$ This scheme generates an ensemble of trajectories between the initial and final states using Monte Carlo sampling in trajectory space. TPS only requires an order parameter to distinguish the initial and final states; this order parameter does not need to be the true reaction coordinate. TPS thus makes it possible to compute the rate constant without prior knowledge of the reaction mechanism. However, this method does require knowledge of the steady-state phase-space distribution, which is needed for the acceptance/rejection step in the Monte Carlo scheme, and it does not allow direct computation of the free-energy barrier separating the two states. Moroni et al. have developed a related method, transition interface sampling (TIS), which relies on the computation of crossing probabilities of a series of interfaces between the initial and final states. ${ }^{9,10,16,17} \mathrm{~A}$ variant of this method, partial path TIS (PPTIS), assumes loss of time correlations in the transition paths over a distance of two interfaces. Moroni et al. have recently shown how the free-energy barrier as well as the rate constant can be obtained from a single TIS/PPTIS calculation. ${ }^{18}$ As for TPS, both TIS and PPTIS require the system to be in thermodynamic equilibrium. The "mile-stoning" method of Faradjian and Elber also employs a series of interfaces to compute rate constants and also assumes that the interfacecrossing probability does not depend upon the full history of the path. ${ }^{19}$ In an alternative approach, Vanden-Eijnden et al. have developed a set of "string" methods, which can be used to compute minimum free-energy paths and the probability current of reactive trajectories for equilibrium systems. ${ }^{20,21}$

The algorithms discussed above-TPS, (PP)TIS, mile stoning, and the string methods-are limited to systems that are in thermodynamic equilibrium. The FFS method and its variants were developed to calculate rate constants and transition paths for rare events in equilibrium and nonequilibrium systems with stochastic dynamics. ${ }^{11-13}$ Like TIS, PPTIS, and mile stoning, FFS uses a series of interfaces to compute the rate constant. However (unlike PPTIS and milestoning), FFS does not make the Markovian assumption that the distribution of paths at the interfaces is independent of the path histories. The order parameter that is used to define the location of the interfaces need not be the reaction coordinate, and the choice of order parameter, in principle, does not bias the dynamics of the transition paths.

We have recently shown that the rate constant for activated processes in nonequilibrium systems that are in steady state can also be written in the form of Eq. (1). ${ }^{22}$ The quantity $\rho(q)$ is then the stationary probability distribution function for the order parameter $q$. In this paper we show that the stationary distribution $\rho(q)$, as well as the forward and backward rate constants and transition paths, can be obtained by performing two FFS calculations: one for the transition from the initial to the final state, and the other for the reverse transition. The method can be applied to both nonequilibrium and equilibrium systems; in the latter case, $\rho(q)$ corresponds to the Boltzmann distribution. The method is conceptually similar to that used in TIS and PPTIS to compute free-energy barriers, in the sense that the stationary distribution $\rho(q)$ is obtained by matching the forward and backward trajectories. $9,10,16,17$

In the next section, we explain the FFS algorithm. ${ }^{11}$ In Secs. III and IV, we discuss the theory and method for obtaining stationary distributions. We then illustrate the method using symmetric and asymmetric double-well potentials (Sec. VI), and the two-dimensional nonequilibrium rare event problem proposed by Maier and Stein (Sec. VII). In Sec. VIII, we use the method to calculate the free-energy barrier for nucleation in a two dimensional Ising system. Finally, in Sec. IX, we compute nonequilibrium stationary probability distributions for a bistable model genetic switch.

\section{FORWARD FLUX SAMPLING}

We consider rare, spontaneous transitions between two regions of state space $A$ and $B$. The phase-space coordinates are denoted by $x$ and the regions $A$ and $B$ are defined in terms of an order parameter $\lambda(x)$ such that the system is in state $A$ if $\lambda(x)<\lambda_{0}$, and it is in state $B$ if $\lambda(x)>\lambda_{n}$. The key principle is to use a series of interfaces $\lambda_{0}, \lambda_{1}, \ldots, \lambda_{n-1}, \lambda_{n}$ to drive the system from state $A$ to state $B$ in a ratchet-like manner. The idea of the interfaces is that they make it possible to capitalize on all those fluctuations that bring the system in the direction of the final state $B$.

Supposing that with a conventional [say molecular dynamics (MD)] simulation, the system exhibits a rare fluctuation that moves it up the barrier, and that it crosses an interface between state $A$ and the top of the barrier, if we would continue this successful run, then most likely the system would roll back down the hill, i.e., relax back towards state $A$, and one would have to wait for "another" rare fluctuation that moves the system in the direction of $B$. By storing the configurations at the interfaces, we can thus efficiently exploit all those fluctuations that move the system up the barrier.

In this paper, we make use of the original FFS scheme ${ }^{11}$ presented in more details in Ref. 12.

In FFS, one first performs a conventional, brute-force 
simulation in state $A$. Each time the system crosses the interface $\lambda_{0}$ in the direction of increasing $\lambda$ during this simulation, the coordinates of that state point are stored. One also measures the average number per unit time of these crossings. At the end of this simulation, one has a measure of the flux $\Phi_{A}$ of trajectories crossing $\lambda_{0}$ from $A$, as well as a collection of state points corresponding to crossings of the first interface, $\lambda_{0}$, coming from $A$. This collection is then used to provide starting points for a set of trajectories, each of which is continued until the system either reaches the next interface, $\lambda_{1}$, or returns to state $A$ (i.e., reaches $\lambda_{0}$ ). This procedure generates a new collection of state points at the next interface, which are the end points of those trajectories that arrived at $\lambda_{1}$ from $\lambda_{0}$. One also obtains an estimate of the probability $P\left(\lambda_{1} \mid \lambda_{0}\right)$ that a trajectory which reaches $\lambda_{0}$ from $A$ will subsequently reach $\lambda_{1}$ without returning to $A$ this is simply the fraction of trajectories which arrive at $\lambda_{1}$. By repeating this procedure for all subsequent interfaces, one has for each interface $i$ an estimate of the probability $P\left(\lambda_{i+1} \mid \lambda_{i}\right)$ that, given that a trajectory has reached interface $i$ coming from $A$, it subsequently reaches $\lambda_{i+1}$ before returning to $A$. The rate constant $k_{A B}$ can then be obtained from ${ }^{18}$

$$
k_{A B}=\Phi_{A} \prod_{i=0}^{n-1} P\left(\lambda_{i+1} \mid \lambda_{i}\right) .
$$

By tracing back paths that successfully arrive at $\lambda_{n}$, one can also sample the transition path ensemble for the rare event. Analysis of these paths can lead to insight into the mechanism by which the event occurs.

\section{STATIONARY DISTRIBUTIONS: THEORY}

We are interested in computing the stationary distribution $\rho(q)$, where $\rho(q) d q$ is the probability of observing the order parameter $q$ in the range $q \rightarrow q+d q$, for a system that is in a stationary state. We stress the fact that the order parameter $q$ for the computation of the stationary distribution function need not be the same as the order parameter $\lambda$ that is chosen for the FFS calculation. The stationary distribution can be expressed as

$$
\rho(q)=\langle\delta(q-q(x))\rangle,
$$

where $x$ is a point in the multidimensional phase-space. For equilibrium systems, the contributions to the average in Eq. (3) are weighted according to the Boltzmann distribution, while for nonequilibrium systems they are weighed according to the steady-state phase-space density. For both equilibrium and nonequilibrium systems that are in steady-state and ergodic, this ensemble average is equivalent to a time average over a long brute-force simulation, in which $\rho(q)$ measures the frequency with which value $q$ of the order parameter is "visited" by the trajectory.

The distribution function $\rho(q)$ is easy to sample close to the stable states $A$ and $B$, using conventional, brute-force simulation. However, this method will lead to poor statistics in the "barrier" region between $A$ and $B$, which is rarely visited. We use FFS to obtain $\rho(q)$ in the barrier region, and supplement this with conventional sampling in the two stable states to obtain the complete distribution function.

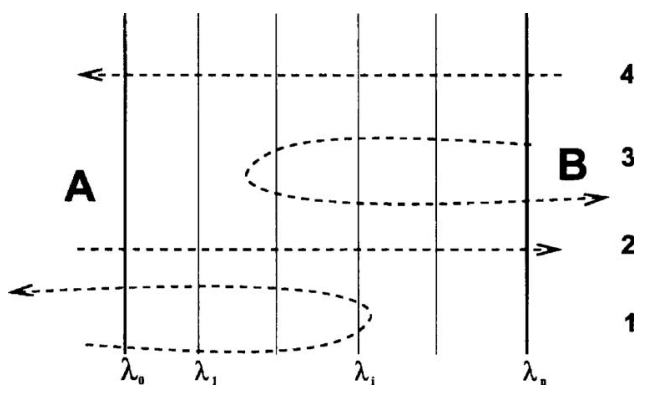

FIG. 1. Sketch of all the possible trajectories that contribute to the stationary distribution $\rho(q)$ [see Eq. (3)]: Trajectory 1 comes from $A$ and goes back to $A$; trajectory 2 comes from $A$ and goes to $B$; trajectory 3 comes from $B$ and goes back to $B$; trajectory 4 comes from $B$ and goes to $A$. The FFS simulation from $A$ to $B$ harvests the trajectories corresponding to types 1 and 2 , while the FFS simulation for the reverse transition generates trajectories of types 3 and 4 . The interfaces $\left\{\lambda_{0}, \lambda_{1}, \ldots, \lambda_{n-1}, \lambda_{n}\right\}$ used in the FFS simulation are also shown.

The key idea which we use to obtain stationary distributions with FFS is to divide the "visits" of an imaginary, very long simulation trajectory to value $q$ of the order parameter into two categories, according to whether the trajectory was most recently in state $A$ or state $B$. We therefore write $\rho(q)$ as the sum of two contributions,

$$
\rho(q)=\psi_{A}(q)+\psi_{B}(q),
$$

where $\psi_{A}(q)$ is the contribution to the probability density $\rho(q)$ from those trajectories that come from region $A$, and $\psi_{B}(q)$ is the contribution due to trajectories coming from $B$ (see Fig. 1). In the basins of attraction $A$ and $B$, the trajectories will quickly loose memory of where they came from, i.e., we expect excursions out of a basin of attraction to be uncorrelated. This, as we describe below, makes it possible to obtain the distribution function $\psi_{A}(q)$ from a FFS simulation for the transition from $A$ to $B$, while $\psi_{B}(q)$ can be computed using a FFS simulation for the reverse transition (see Fig. 1). For equilibrium systems, the free energy profile can be obtained from $\Delta G \sim-k_{B} T \ln [\rho(q)]$ once $\rho(q)$ is known.

The function $\psi_{A}(q)$ is given by

$$
\psi_{A}(q)=p_{A} \Phi_{A} \tau_{+}\left(q ; \lambda_{0}\right) .
$$

Here, $p_{A}$ is the probability that the system is in state $A$ and $\Phi_{A}$ is, as in Eq. (2), the flux of trajectories leaving state $A$ (i.e., crossing the surface $\lambda_{0}$ coming from $A$ ). The quantity $\tau_{+}\left(q ; \lambda_{0}\right) \equiv\langle\delta(q-q(x))\rangle_{\lambda_{0}}$ is the average time spent at order parameter $q$ by a trajectory that originates from interface $\lambda_{0}$. We note that $\tau_{+}\left(q ; \lambda_{0}\right)$ includes contributions both from paths that start in $A$ and ultimately reach $B$, and from those that start in $A$ and ultimately return to $A$ without reaching $B$ (see Fig. 1).

In FFS, we use a series of interfaces to sample the phase space between $A$ and $B$ in stages. At each stage, an ensemble of paths is generated by firing off trajectories from points on an interface that have been obtained in the previous stage; each of these trajectories is terminated as soon as it reaches either the next interface or $\lambda_{0}$ (see Sec. II). We denote the average time spent at order parameter $q$, for a trial run that is 
fired from interface $\lambda_{i}$ (and terminated at $\lambda_{i+1}$ or $\lambda_{0}$ ) in the FFS procedure, by $\pi_{+}\left(q ; \lambda_{i}\right)$. As shown in the Appendix, $\tau_{+}\left(q ; \lambda_{0}\right)$ is then given by

$$
\tau_{+}\left(q ; \lambda_{0}\right)=\tau_{+}\left(q ; \lambda_{0}\right)+\sum_{i=1}^{n-1} \pi_{+}\left(q ; \lambda_{i}\right) \prod_{j=0}^{i-1} P\left(\lambda_{j+1} \mid \lambda_{j}\right) .
$$

The factor $\Pi_{j=0}^{i-1} P\left(\lambda_{j+1} \mid \lambda_{j}\right)$ reweighs the distribution $\pi\left(q ; \lambda_{i}\right)$ to correct for the enhanced sampling at interface $i$ which has been achieved by the FFS procedure. This factor is a direct output from the FFS simulation (see Sec. II, Eq. (2), and the Appendix). The FFS calculation for the forward transition thus yields $k_{A B}, \Phi_{A}$, and $\tau_{+}\left(q ; \lambda_{0}\right)$.

To calculate $\rho(q)$, we also need to evaluate $\psi_{B}(q)$ in Eq. (4), by carrying out a FFS calculation in the reverse direction, from $B$ to $A$. The entire FFS algorithm is carried out in reverse: in the initial, brute-force simulation, we begin with the system in state $B$ and collect crossings of interface $\lambda_{n}$ coming from $B$. We fire trajectories from $\lambda_{i}$ which either reach $\lambda_{i-1}$ or return to $\lambda_{n}$. The result is a value for the reverse rate constant $k_{B A}$, the flux $\Phi_{B}$, and the distribution functions $\pi_{-}\left(q ; \lambda_{i}\right)$ for the order parameter $q$, sampled over the ensemble of paths that are fired from interface $\lambda_{i}$ and terminated at $\lambda_{i-1}$ or $\lambda_{n}$ in the reverse FFS procedure. These are related to the distribution function $\tau_{-}\left(q ; \lambda_{n}\right)$ for all trajectories leaving $\lambda_{n}$ from $B$ by

$$
\tau_{-}\left(q ; \lambda_{n}\right)=\pi_{-}\left(q ; \lambda_{n}\right)+\sum_{i=n-1}^{1} \pi_{-}\left(q ; \lambda_{i}\right) \prod_{j=n}^{i+1} P\left(\lambda_{j-1} \mid \lambda_{j}\right),
$$

where $P\left(\lambda_{j-1} \mid \lambda_{j}\right)$ are the conditional probabilities of reaching interface $j-1$ from $\lambda_{j}$, evaluated in the reverse FFS procedure. The distribution $\psi_{B}(q)$ is then given by

$$
\psi_{B}(q)=p_{B} \Phi_{B} \tau_{-}\left(q ; \lambda_{n}\right) .
$$

To obtain $p_{A}$ and $p_{B}$ in Eqs. (5) and (8), we note that in steady-state

$$
p_{A} k_{A B}=p_{B} k_{B A},
$$

where $k_{A B}$ and $k_{B A}$ are the forward and backward rate constants measured in the forward and backward FFS calculations, respectively. Since we are assuming a two-state system (i.e., ignoring intermediate states), we also know that $p_{A}$ $+p_{B}=1$. This implies that

$$
p_{A}=\frac{k_{B A} / k_{A B}}{1+k_{B A} / k_{A B}}
$$

and

$$
p_{B}=\frac{1}{1+k_{B A} / k_{A B}} .
$$

Combining all this information and using Eq. (4), we can obtain the stationary distribution function $\rho(q)$ in the region $\lambda_{0}<\lambda<\lambda_{n}$. This can be combined with brute-force sampling in the $A$ and $B$ basins to determine $\rho(q)$ over the full range of $q$ values, if required.

\section{STATIONARY DISTRIBUTIONS: METHOD}

As discussed above, to obtain the stationary distribution $\rho(q)$ in the region $\lambda_{0}<\lambda<\lambda_{n}$ we perform one FFS simulation for the transition from $A$ to $B$ and one for the reverse transition. For details on the implementation of the FFS method to compute the fluxes $\Phi_{A}$ and $\Phi_{B}$, as well as the rate constants $k_{A B}$ and $k_{B A}$, we refer to Ref. 11 . Here, we briefly discuss how $\tau_{+}\left(q ; \lambda_{0}\right)$ and $\tau_{-}\left(q ; \lambda_{n}\right)$ are obtained in practice. We consider $\tau_{+}\left(q ; \lambda_{0}\right) ; \tau_{-}\left(q ; \lambda_{n}\right)$ is obtained similarly, but in reverse, as described above. Our aim is to calculate the quantities $\pi_{+}\left(q ; \lambda_{i}\right)$ and $P\left(\lambda_{j+1} \mid \lambda_{j}\right)$ in Eq. (6) [or alternatively for the reverse transition, $\pi_{-}\left(q ; \lambda_{i}\right)$ and $P\left(\lambda_{j-1} \mid \lambda_{j}\right)$ in Eq. (7)]. Considering only the forward FFS procedure: at each interface $\lambda_{i}$ we fire a total of $M_{i}$ trial runs, each of which is terminated when the system reaches either $\lambda_{i+1}$ or $\lambda_{0}$. The probability $P\left(\lambda_{i+1} \mid \lambda_{i}\right)$ is then estimated as

$$
P\left(\lambda_{i+1} \mid \lambda_{i}\right)=\frac{N_{i}^{\mathrm{s}}}{M_{i}},
$$

where $N_{i}^{\mathrm{s}}$ is the number of trials that have successfully reached $\lambda_{i+1}$. The function $\pi_{+}\left(q ; \lambda_{i}\right)$ is given by

$$
\pi_{+}\left(q ; \lambda_{i}\right)=\frac{N_{q}}{\Delta q M_{i}},
$$

where $N_{q}$ is the number of times that during this set of trial runs the order parameter of the system has a value between $q$ and $q+\Delta q$. This is given by $N_{q}=\Delta t \sum_{k=0}^{M_{i}} \sum_{s=0}^{n_{k}} h_{q}\left(x_{k, s}\right)$, where the double sum runs over all the $n_{k}$ steps of all the $M_{i}$ trial paths starting at interface $\lambda_{i}$ and $h_{q}(x)$ is an indicator function that is one if during a time step the system is between $q$ and $q+\Delta q$, and zero otherwise; again, note that $n_{k}$ varies from one path to the next. The simulation time step $\Delta t$ can, in fact, be neglected, since is it a constant and we plan to normalize $\rho(q)$ in any case. For algorithms in which the time step can vary, $N_{q}$ is given by $N_{q}=\sum_{k=0}^{M_{i}} \sum_{s=0}^{n_{k}} \Delta t_{k, s} h_{q}\left(x_{k, s}\right)$, where $\Delta t_{k, i}$ is the magnitude of time step $s$ of path $k$. To obtain $\tau_{+}\left(q ; \lambda_{0}\right)$ we reweigh $\pi_{+}\left(q ; \lambda_{i}\right)$ and sum over all interfaces using Eq. (6). Once $\psi_{A}(q)$ and $\psi_{B}(q)$ have been obtained by performing FFS simulations in both directions, $\rho(q)$ can be obtained via Eq. (3). We note that $\psi_{A}(q)$ and $\psi_{B}(q)$ should not be individually normalized, since they are not probability distribution functions in their own right, but simply contributions to the distribution function $\rho(q)$. If the average path length for paths originating in $A$ and $B$ is different, then the integral of $\psi_{A}(q)$ and $\psi_{B}(q)$ over $q$ will be different. Normalizing $\psi_{A}(q)$ and $\psi_{B}(q)$ will result in incorrect relative contributions to $\rho(q)$ from trajectories originating in $A$ and in $B$. We also note that, when evaluating $N_{q}$, it is important not to double count the start and end points of trial runs; if the initial point of a trial run is deemed to count towards the $N_{q}$ histogram for that interface, then the final point should not count as it will be counted as an initial point in the histogram for the next interface.

The above procedure generates $\rho(q)$ in the region $\lambda_{0}$ $<\lambda<\lambda_{n}$. To obtain the full distribution $\rho(q)$, we sample using conventional, brute-force simulation the steady-state distribution for the order parameter $q$ in the $A$ and $B$ regions. This will result in distributions for $\lambda<\lambda_{0}+\Delta \lambda$ ( $A$ region) 
and $\lambda>\lambda_{n}-\Delta \lambda$ ( $B$ region), where $\Delta \lambda$ is a small overlap. An easy way to fit these curves together is to take their logarithms: the three overlapping parts for $\log \rho(q)$ can then be fitted together by a least squares fitting procedure (since a constant may be added to each without affecting the distribution). The resulting full profile $\rho(q)$ is obtained by exponentiation $\log \rho(q)$, and the stationary probability distribution can finally be normalized.

\section{STATIONARY DISTRIBUTIONS OF MULTIPLE ORDER PARAMETERS}

It is important to point out that the procedure described in Sec. IV may be adapted to allow the computation of stationary distribution functions of several order parameters ("free energy landscapes" in the equilibrium case). In the case where we wish to find the stationary distribution (for $\lambda_{0}<\lambda<\lambda_{n}$ ) as a function of two order parameters $q$ and $r$, Eq. (4) is replaced by

$$
\rho(q, r)=\psi_{A}(q, r)+\psi_{B}(q, r),
$$

where

$$
\begin{aligned}
& \psi_{A}(q, r)=p_{A} \Phi_{A} \tau_{+}\left(q, r ; \lambda_{0}\right), \\
& \psi_{B}(q, r)=p_{B} \Phi_{B} \tau_{-}\left(q, r ; \lambda_{n}\right)
\end{aligned}
$$

and

$$
\begin{aligned}
& \tau_{+}\left(q, r ; \lambda_{0}\right)=\pi_{+}\left(q, r ; \lambda_{0}\right)+\sum_{i=1}^{n-1} \pi_{+}\left(q, r ; \lambda_{i}\right) \prod_{j=0}^{i-1} P\left(\lambda_{j+1} \mid \lambda_{j}\right), \\
& \tau_{-}\left(q, r ; \lambda_{n}\right)=\pi_{-}\left(q, r ; \lambda_{n}\right)+\sum_{i=n-1}^{1} \pi_{-}\left(q, r ; \lambda_{i}\right) \prod_{j=n}^{i+1} P\left(\lambda_{j-1} \mid \lambda_{j}\right) .
\end{aligned}
$$

To evaluate the functions $\pi_{+}\left(q, r ; \lambda_{i}\right)$ and $\pi_{-}\left(q, r ; \lambda_{i}\right)$, we use a two-dimensional histogram $N_{q r}$ in the coordinates $q$ and $r$,

$$
\pi_{+}\left(q, r ; \lambda_{i}\right)=\frac{\Delta t N_{q r}}{\Delta q \Delta r M_{i}}
$$

and the equivalent for the reverse FFS procedure. Here, $N_{q r}$ is the number of time steps during the set of $M_{i}$ trial runs fired from $\lambda_{i}$ for which the system has a value of $q$ between $q$ and $q+\Delta q$ and a value of $r$ between $r$ and $r+\Delta r$.

\section{TESTING ON A ONE-DIMENSIONAL SYSTEM}

As an initial test, we have applied the method to a single particle moving with Brownian dynamics in a onedimensional double-well potential,

$$
V(x)=-b x^{2}+c x^{4},
$$

with $b=2$ and $c=1$. Distance is measured here in units of $x_{0}$, while time is measured in units of $t_{0}$. The stationary distribution function, as a function of the $x$ coordinate, is the Boltzmann distribution,
TABLE I. Interfaces and the number of trials at each interface for the FFS sampling of the symmetric one dimensional double-well potential.

\begin{tabular}{cccccc}
\hline \hline$i$ & $\lambda_{i}$ & $M\left(\lambda_{i}\right)$ & $i$ & $\lambda_{i}$ & $M\left(\lambda_{i}\right)$ \\
\hline 0 & -0.8 & 100000 & 4 & -0.1 & 25000 \\
1 & -0.7 & 250000 & 5 & 0.1 & 12000 \\
2 & -0.5 & 17000 & 6 & 0.3 & 10000 \\
3 & -0.3 & 70000 & 7 & 0.5 & 10000 \\
\hline \hline
\end{tabular}

$$
\rho(x) \sim e^{-V(x) / k_{B} T} .
$$

The system is symmetric, so that $p(A)=p(B)$. The particle moves according to

$$
v(t)=\frac{D}{k_{B} T} f(t)+\xi(t),
$$

where $f$ is the instantaneous force, $D$ is the diffusion constant, and $\xi$ is chosen at random from a Gaussian distribution with zero mean and variance $\left\langle\xi^{2}\right\rangle=2 D d t .{ }^{23}$ We used the following values: $D=0.01 x_{0}^{2} / t_{0}, k_{B} T=0.1$, and $d t=0.05 t_{0}$. We have carried out FFS simulations with $n=8$ interfaces, $N_{1}$ $=10000$ points at interface $\lambda_{0}$, and parameters as shown in Table I. We obtained a forward rate constant $k_{A B}$ $=3.87 \pm 0.05 \times 10^{-6} t_{0}^{-1}$ (repeating twice to obtain error bar). Because of the symmetry of the problem, it was not necessary to carry out separate FFS calculations for the forward and backward transitions in this case; the backward probability distribution can be obtained from the forward one by a simple coordinate inversion. The stationary distribution obtained from the FFS calculation is compared to the expected Boltzmann distribution in Fig. 2.

We have also considered the asymmetric case, in which a term linear in $x$ is included in Eq. (18),

$$
V(x)=a x+-b x^{2}+c x^{4},
$$

with $a=0.25, b=2, c=1, D=0.01 x_{0}^{2} / t_{0}, k_{B} T=0.1$, and $d t$ $=0.05 t_{0}$. In this case, $p(A) \neq p(B)$ and it is necessary to carry out FFS sampling in both directions. We carry out FFS simulations, again with $n=8$ interfaces and $N_{1}=10000$. For the forward transition, we used $\lambda=x$, and for the backward transition, $\lambda=-x$. For both the forward and backward transitions, the parameters for the FFS runs were as shown in Table II. The forward and backward rate constants were calculated to

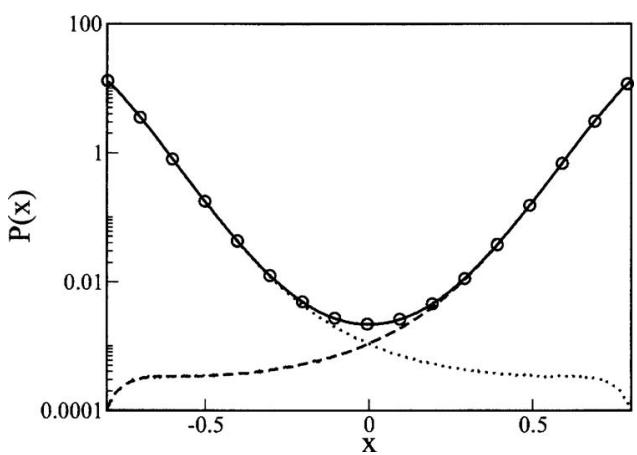

FIG. 2. Stationary distribution (solid line) obtained using the procedure described above, compared to the normalized Boltzmann distribution (circles) for a symmetric double well potential. The dotted and dashed lines show $\psi_{A}(x)$ and $\psi_{B}(x)$, respectively. 
TABLE II. Interfaces and the number of trials at each interface for the FFS sampling of the asymmetric one dimensional double-well potential.

\begin{tabular}{cccccc}
\hline \hline$i$ & $\lambda_{i}$ & $M\left(\lambda_{i}\right)$ & $i$ & $\lambda_{i}$ & $M\left(\lambda_{i}\right)$ \\
\hline 0 & -0.8 & 100000 & 4 & -0.1 & 50000 \\
1 & -0.7 & 560000 & 5 & 0.1 & 20000 \\
2 & -0.5 & 430000 & 6 & 0.3 & 12000 \\
3 & -0.3 & 170000 & 7 & 0.5 & 10000 \\
\hline \hline
\end{tabular}

be $k_{A B}=3.03 \pm 0.06 \times 10^{-7} t_{0}^{-1}$ and $k_{B A}=3.96 \pm 0.03 \times 10^{-5} t_{0}^{-1}$, and the fluxes across the $A$ boundary were $\Phi_{A}$ $=0.1526 \pm 0.0007 t_{0}^{-1}$ and $\Phi_{B}=0.3648 \pm 0.0001 t_{0}^{-1}$, respectively. Figure $3(\mathrm{a})$ shows $\tau_{+}\left(x ; \lambda_{0}\right)$ and $\tau_{-}\left(x ; \lambda_{n}\right)$, while Fig. 3(b) shows $\rho(x)$, calculated from Eq. (3) and normalized. Excellent agreement was obtained with the expected Boltzmann distribution.

\section{TESTING ON THE TWO-DIMENSIONAL MAIER-STEIN SYSTEM}

We now move on to demonstrate the calculation of twodimensional stationary distributions using a rare event problem in two dimensions that may be in or out of equilibrium-overdamped Brownian motion in the force field proposed by Maier and Stein, ${ }^{24-26}$

$$
\begin{aligned}
& \dot{x}=f_{x}(\mathbf{x}, t)+\xi_{x}(t), \\
& \dot{y}=f_{y}(\mathbf{x}, t)+\xi_{y}(t),
\end{aligned}
$$

where $\mathbf{x}=(x, y)$. The force field $\mathbf{f}=\left(f_{x}, f_{y}\right)$ (which is time independent) is given by

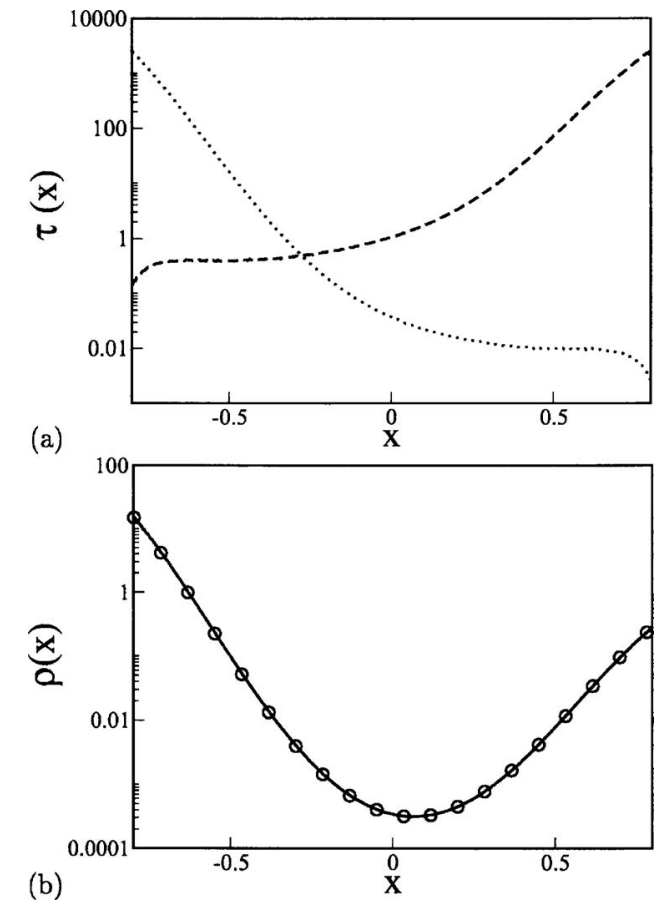

FIG. 3. Asymmetric double well potential (a): $\tau_{+}\left(x ; \lambda_{0}\right) / \Delta t$ (dotted line) and $\tau_{-}\left(x ; \lambda_{n}\right) / \Delta t$ (dashed line). (b) Final result for $\rho(x)$ obtained from Eq. (3) (solid line) compared to the expected Boltzmann distribution (circles).
TABLE III. Interfaces and the number of trials per interface for the MaierStein system.

\begin{tabular}{cccccc}
\hline \hline$i$ & $\lambda_{i}$ & $M\left(\lambda_{i}\right)$ & $i$ & $\lambda_{i}$ & $M\left(\lambda_{i}\right)$ \\
\hline 0 & -0.8 & 1000000 & 4 & 0.2 & 200000 \\
1 & -0.6 & 500000 & 5 & 0.4 & 120000 \\
2 & -0.4 & 300000 & 6 & 0.6 & 100000 \\
3 & -0.2 & 250000 & 7 & 0.8 & 100000 \\
\hline \hline
\end{tabular}

$$
\begin{aligned}
& f_{x}=x-x^{3}-\alpha x y^{2}, \\
& f_{y}=-\mu y\left(1+x^{2}\right)
\end{aligned}
$$

and the stochastic force $\xi=\left(\xi_{x}, \xi_{y}\right)$ results from $\delta$-function-correlated white noise with variance $\epsilon$, such that

$$
\left\langle\xi_{i}(t)\right\rangle=0, \quad\left\langle\xi_{i}(t) \xi_{j}(0)\right\rangle=\epsilon \delta_{i j}, \delta t
$$

where $i=x, y$. This system is bistable, with stable points at $( \pm 1,0)$ and a saddle point at $(0,0)$. When $\alpha=\mu$, the force field can be expressed as the gradient of a potential energy function and the system can be considered to be "at equilibrium," while when $\alpha \neq \mu$, the force field $\mathbf{f}$ cannot be expressed as the gradient of a potential and the system is thus intrinsically nonequilibrium. In these simulations, we use $\epsilon$ $=0.1$. Taking $\lambda=x$, we follow the procedure described in Sec. $\mathrm{V}$ to calculate the stationary distribution for $-0.8<x<0.8$ as a function of the two order parameters $x$ and $y$. For the FFS calculations, we use eight interfaces, $\lambda_{0}=-0.8$ and $\lambda_{7}=0.8$, and $N_{1}=100000$ initial configurations at $\lambda_{0}$. The parameters used are listed in Table III.

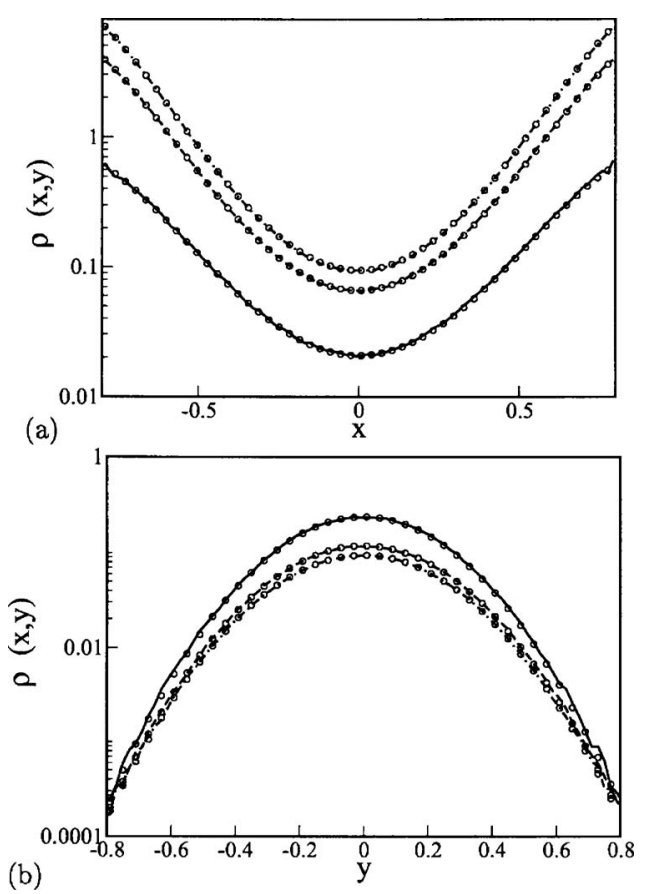

FIG. 4. (a) Computed stationary distribution $\rho(x, y)$ as a function of $x$ for $y=-0.39$ (solid line), $y=-0.19$ (dashed line), and $y=0.01$ (dot-dashed line), compared to the expected Boltzmann distribution (indicated by circles) for the Maier-Stein system with $\epsilon=0.1$ and $\alpha=\mu=1$. (b) $\rho(x, y)$ as a function of $y$ for $x=-0.312$ (solid line), $x=-0.152$ (dashed line), and $x=0.008$ (dotdashed line). 


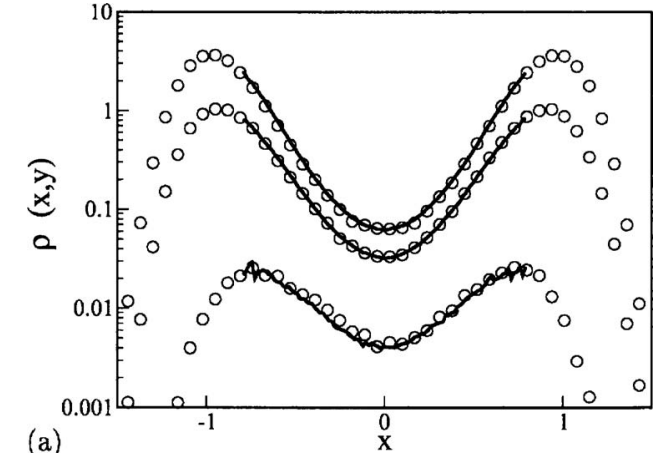

(a)

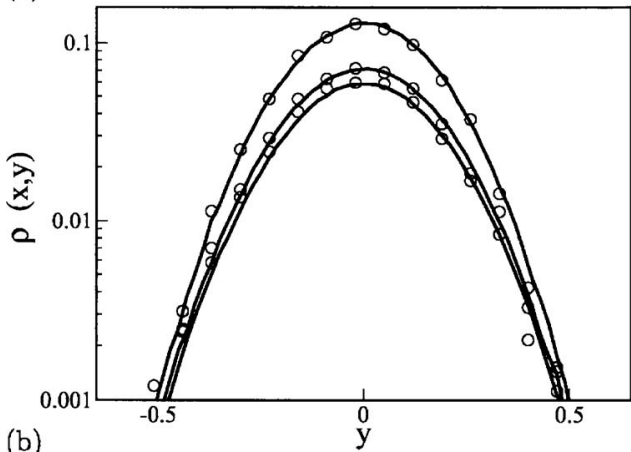

(b)

FIG. 5. $\rho(x, y)$ for the Maier-Stein system with $\alpha=6.67, \mu=2$, and $\epsilon=0.1$. (a) $\rho(x, y)$ as a function of $x$ for $y=-0.39$ (solid line), $y=-0.19$ (dashed line), and $y=0.01$ (dot-dashed line). (b) $\rho(x, y)$ as a function of $y$ for $x=-0.312$ (solid line), $x=-0.152$ (dashed line), and $x=0.008$ (dot-dashed line). The results of long brute-force simulations are indicated by circles.

We initially consider an equilibrium case, with $\alpha=\mu=1$. In this case, the particle moves in the potential field $\phi(x, y)$ $=\left[y^{2}\left(1+x^{2}\right) / 2\right]-\left(x^{2} / 2\right)+\left(x^{4} / 4\right)$. Figures $4(\mathrm{a})$ and 4(b) show the stationary distribution $\rho(x, y)$, as a function of $x$ for $y=-0.39, y=-0.19$, and $y=0.01$, and as a function of $y$ for $x=-0.312, x=-0.152$, and $x=0.008$. In both panels, the results are in excellent agreement with the expected Boltzmann distribution (shown by circles).

We next discuss the nonequilibrium case $(\alpha \neq \mu)$, taking $\alpha=6.67, \mu=2.0$, and $\epsilon=0.1$. Figure 5 shows equivalent results to Fig. 4, but this time the FFS results are compared to stationary distributions computed from long brute-force simulations. The brute-force simulation results are normalized over all space; the FFS results are multiplied by a constant scaling factor to bring them into agreement since they are $a$ priori normalized over the region $-0.8<x<0.8$ only. Very good agreement is observed.

\section{HOMOGENEOUS NUCLEATION IN A TWO-DIMENSIONAL ISING MODEL}

We now address a rare event problem in a more complex system: homogeneous nucleation in a two-dimensional Ising model. For now, we confine ourselves to an equilibrium system without any external shear; nonequilibrium nucleation in an Ising model with an external shearing field will be considered in future work. ${ }^{27}$ The two-dimensional Ising model consists of an $L \times L$ square lattice of spins with nearest neighbor interactions and periodic boundary conditions. Its Hamiltonian $^{28}$

$$
H=-J \sum_{i j}^{\prime} \sigma_{i} \sigma_{j}-h \sum_{i} \sigma_{i}
$$

where $J$ is the coupling constant between neighboring spins $\left(\sigma_{i}= \pm 1\right)$ and $h$ the external magnetic field. The prime indicates a sum over first nearest neighbor interactions only. We simulate a system with $N=45 \times 45=2025$ spins, a positive magnetic field $\beta h=0.05$, and a positive coupling constant $\beta J=0.65$, above the critical coupling $J_{c}$. The thermodynamically stable state is therefore a ferromagnetic one with net positive magnetization, meaning that the system tends to have the majority of its spins in the "up state." However, the state with an overall negative magnetization (i.e., spins predominantly in the down state) is metastable and the system will remain in that state for a significant time if initialized with predominantly down spins. We aim to compute the freeenergy barrier, as well as the rate constant, for transitions from the metastable "down state" to the thermodynamically stable "up state." We begin our simulations in the down state and consider the formation of a cluster of up spins, under conditions of moderate supersaturation (these conditions are identical to those used by $\mathrm{Sear}^{29}$ ). All of our simulations are performed using a Metropolis Monte Carlo algorithm, in which we attempt to flip each spin once, on average, during each Monte Carlo cycle.

According to classical nucleation theory, ${ }^{30}$ the free energy cost of forming a square nucleus of edge length $L$ is given by the sum of a line energy and a surface energy,

$$
\Delta G=4 \gamma L-2 h L^{2},
$$

where $\gamma$ is the interfacial free energy, $h$ is the driving force for nucleation (magnetic field), and $-2 h L^{2}$ is the energy cost of flipping the whole square nucleus with area $L^{2}$. Using Eq. (26), the nucleation free energy barrier height is given by

$$
\Delta G^{*}=\frac{2 \gamma^{2}}{h} .
$$

Plugging in numbers, if we take the interfacial free energy to be $\beta \gamma=0.74,{ }^{29,31}$ the barrier height as predicted by classical nucleation theory is $\beta \Delta G^{*} \sim 22$.

We have computed the nucleation free energy barrier using two simulation techniques: umbrella sampling ${ }^{1-4}$ and FFS. In both cases, we characterize the extent of the transition using a global order parameter, $q \equiv S$, the total number of up spins in the system. The free-energy barrier is then defined as $\beta \Delta G(S) \equiv-\ln [\rho(S) / N]$, where $\rho(S)$ is the probability of observing $S$ up spins in the stationary state.

For our umbrella sampling calculations, we use a series of "windows," defined by a harmonic potential in $S$, to bias the sampling of phase space. ${ }^{1-4}$ We use 25 windows to cover the range $0 \leqslant S \leqslant 300$, with an overlap of 11 between neighboring windows. We sample each window for 500000 Monte Carlo (MC) cycles, and fit the resulting histograms together using a least-squares fitting procedure to obtain the free-energy profile in the range $0 \leqslant S \leqslant 300$. We do not attempt to calculate the barrier for values of $S$ greater than 300 , since once the top of the barrier is crossed, the system is expected to evolve rapidly and we cannot reply on the as- 


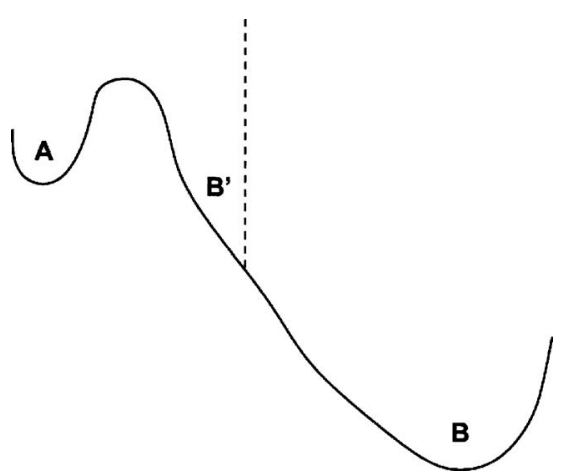

FIG. 6. Schematic view of the free energy landscape. $A$ is the metastable "down state," $B$ is the "real" thermodynamically stable state, and $B$ ' is the "artificial" stable state, constructed by introducing a reflecting wall at $S$ $=S_{B^{\prime}}=1050$

sumption of local thermodynamic equilibrium. Moreover, when $S$ is large, the growing nucleus is likely to interact with its periodic images in neighboring cells, making the results highly system-size dependent.

The interfaces $\lambda_{i}$ for the FFS calculations are also defined in terms of the order parameter $S$. To calculate the free-energy barrier using FFS, we need to be able to sample the reverse transition, from the thermodynamically stable up state to the metastable down state. In general, this is very difficult for a nucleation problem, since the thermodynamic state is much more stable than the metastable state and there is a very high free-energy barrier for the system to return to the down state, making the reverse transition difficult to sample, even with FFS. We have overcome this problem in this case by constructing a reflecting wall beyond the top of the nucleation barrier. This wall is incorporated via a constraint on the system dynamics: each trial move that leads to $S>S_{B^{\prime}}$ is simply rejected. Since we are only interested in the free-energy profile in the region between $A$ and the top of the barrier, we may perturb the free-energy landscape outside this region as we choose. This fact, which is also exploited in umbrella sampling, depends on the system being in equilibrium; for a driven system, we would not be able to use this approach. The reflecting wall, located at $S=S_{B^{\prime}}=1050$, replaces the $B$ state by an artificial stable state $B^{\prime}$ (see Fig. 6). The free-energy barrier for the $B^{\prime} \rightarrow A$ transition is much lower than that for the $B \rightarrow A$ transition, but the shape of the free-energy barrier on the $A$ side remains unchanged. The use of the reflecting wall greatly facilitates the FFS calculation for the reverse transition; it is possible to carry out the reverse FFS calculations without the wall, but this is rather laborious as it requires a large number of interfaces. We have verified that the location of the reflecting wall is indeed well beyond the top of the free-energy barrier, which is estimated to be at $200<S<280$.

State $A$ is defined by the first interface $\lambda_{0}=30$, i.e., when $0<S<30$ the system is in the $A$ state. State $B^{\prime}$ is defined by $\lambda_{n}=1000$, i.e., when $1000<S<1050$ the system is in the $B^{\prime}$ state. For our FFS calculations, we consider $N_{1}=50$ configurations at the first interface. The interfaces are located, both for the forward and backward sampling, at the values of $S$ given in Table IV, where we also list the number of trials performed at each interface.

The FFS calculation for the forward transition from $A$ to $B^{\prime}$ is straightforward. The flux $\Phi_{A}$ through $\lambda_{0}$ from $A$ is $\Phi_{A}=1.5 \times 10^{-5} \mathrm{MC} \mathrm{step}{ }^{-1} \operatorname{spin}^{-1}$ and the forward rate constant $k_{A B^{\prime}}=2.8 \pm 0.3 \times 10^{-13} \mathrm{MC}$ step $^{-1} \operatorname{spin}^{-1}$ : this is in good agreement with the value of $3.3 \times 10^{-13} \mathrm{MC} \mathrm{step}{ }^{-1}$ spin $^{-1}$ computed for the same system by Sear et al. ${ }^{29}$ The computed forward rate constant does not depend on the reflecting wall position $S_{B^{\prime}}$. This calculation also results in the function $\tau_{+}\left(S ; \lambda_{0}\right)$, as described in Sec. IV. In the reverse direction, we use the same interfaces and sample from $\lambda_{n}$ to $\lambda_{0}$ as described in Sec. IV. We obtain the flux $\Phi_{B}^{\prime}=1.4$ $\times 10^{-6} \mathrm{MC} \mathrm{step}^{-1} \mathrm{spin}^{-1}$ and the backward rate constant $k_{B^{\prime} A}=2.0 \pm 0.2 \times 10^{-19} \mathrm{MC} \mathrm{step}^{-1} \operatorname{spin}^{-1}$. In this procedure, we also compute the function $\tau_{-}\left(S ; \lambda_{n}\right)$, as described in Sec. IV. Combining the rate constants as in Eqs. (10) and (11), we obtain $p_{A}=7 \times 10^{-7}$ and $p_{B^{\prime}}=0.999$. By means of Eqs. (4) and (5) we finally obtain $\rho(S)$ for $30<S<1000$. Fitting this together with the distribution obtained by conventional sampling in state $A$ (as described in Sec. IV), we obtain the free-energy barrier.

Figure 7 shows the results for the nucleation barrier, $\beta \Delta G(S)$, in the range $0<S<300$, computed as $-\ln [\rho(S)] / N$. The free-energy minimum at $S \approx 20$ indicates that for this supersaturation, the system has a small number of up spins even in the down state. The free-energy barriers, as obtained by umbrella sampling and FFS, are $\Delta G^{\mathrm{umbr}}=24.5 k_{B} T$ and $\Delta G^{\mathrm{FFS}}=23 k_{B} T$, respectively. These coincide within the error bars, which for both schemes are on the order of $k_{B} T$. The computed barrier heights also agree remarkably well with the classical nucleation theory (CNT) prediction of $22 k_{B} T$.

TABLE IV. Interfaces and the number of trials per interface for the FFS sampling for the two-dimensional Ising nucleation problem.

\begin{tabular}{ccccccccc}
\hline \hline$i$ & $\lambda_{i}$ & $M_{i}$ & $i$ & $\lambda_{i}$ & $M_{i}$ & $i$ & $\lambda_{i}$ & $M_{i}$ \\
\hline 0 & 30 & 1000 & 9 & 250 & 1000 & 18 & 500 & 1000 \\
1 & 50 & 1000 & 10 & 280 & 1000 & 19 & 550 & 1000 \\
2 & 70 & 1000 & 11 & 300 & 1000 & 20 & 600 & 1000 \\
3 & 100 & 1000 & 12 & 330 & 1000 & 21 & 650 & 1000 \\
4 & 130 & 1000 & 13 & 350 & 1000 & 22 & 700 & 1000 \\
5 & 150 & 1000 & 14 & 380 & 1000 & 23 & 750 & 1000 \\
6 & 180 & 1000 & 15 & 400 & 1000 & 24 & 800 & 1000 \\
7 & 200 & 1000 & 16 & 430 & 1000 & 25 & 850 & 1000 \\
8 & 230 & 1000 & 17 & 450 & 1000 & 26 & 950 & 1000 \\
\hline \hline
\end{tabular}




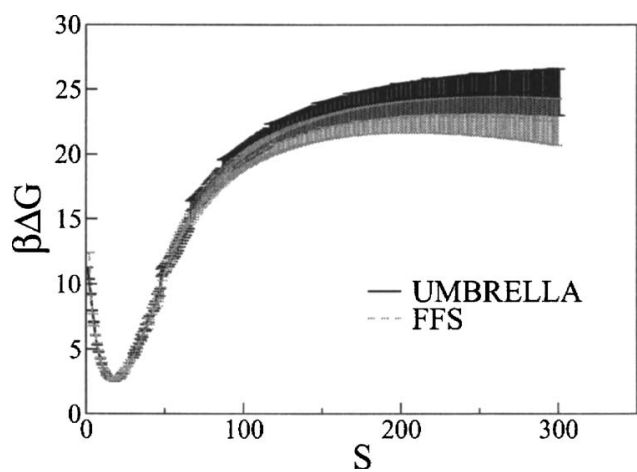

FIG. 7. Free energy barrier for $\beta J=0.65$ and $\beta h=0.05$ calculated using FFS (dashed line; gray) and umbrella sampling (continuous line; black). Error bars are shown for both calculations.

\section{GENETIC SWITCH}

Our final test system is a biologically inspired nonequilibrium rare event problem: a model bistable genetic switch. This is a set of chemical reactions, representing proteinprotein and protein-DNA interactions, as well as protein production and degradation, in a biological cell. The set of reactions shows two stable states, between which the system flips when simulated with stochastic dynamics. This is a particular case of the "exclusive" bistable genetic switch studied by Warren $e t a l .^{22}$ The system does not obey detailed balance, and is therefore out of equilibrium. The set of chemical reactions which we simulate is given in the following with their corresponding rates in parenthesis:

$$
\begin{aligned}
& \mathrm{A}+\mathrm{A} \rightleftharpoons \mathrm{A}_{2} \quad\left(k_{f}, k_{b}\right), \quad \mathrm{B}+\mathrm{B} \rightleftharpoons \mathrm{B}_{2} \quad\left(k_{f}, k_{b}\right), \\
& \mathrm{O}+\mathrm{A}_{2} \rightleftharpoons \mathrm{OA}_{2} \quad\left(k_{\mathrm{on}}, k_{\mathrm{off}}\right), \quad \mathrm{O}+\mathrm{B}_{2} \rightleftharpoons \mathrm{OB}_{2} \quad\left(k_{\mathrm{on}}, k_{\mathrm{off}}\right), \\
& \mathrm{O} \rightarrow \mathrm{O}+\mathrm{A} \quad\left(k_{\text {prod }}\right), \quad \mathrm{O} \rightarrow \mathrm{O}+\mathrm{B} \quad\left(k_{\text {prod }}\right), \\
& \mathrm{OA}_{2} \rightarrow \mathrm{OA}_{2}+\mathrm{A} \quad\left(k_{\text {prod }}\right), \quad \mathrm{OB}_{2} \rightarrow \mathrm{OB}_{2}+\mathrm{B} \quad\left(k_{\text {prod }}\right),
\end{aligned}
$$

$$
\mathrm{A} \rightarrow \varnothing \quad(\mu), \quad \mathrm{B} \rightarrow \varnothing \quad(\mu),
$$

Our model switch is shown schematically in Fig. 8. It consists of two genes, which encode proteins $\mathrm{A}$ and $\mathrm{B}$. Proteins $\mathrm{A}$ and $\mathrm{B}$ can form homodimers $\mathrm{A}_{2}$ and $\mathrm{B}_{2}$, as in Eq. (28a). The production rates for A and $\mathrm{B}$ depend on the state of the DNA sequence $\mathrm{O}$, which is a regulatory site to which either $\mathrm{A}_{2}$ or $\mathrm{B}_{2}$ can bind. When $\mathrm{O}$ is free (not bound by

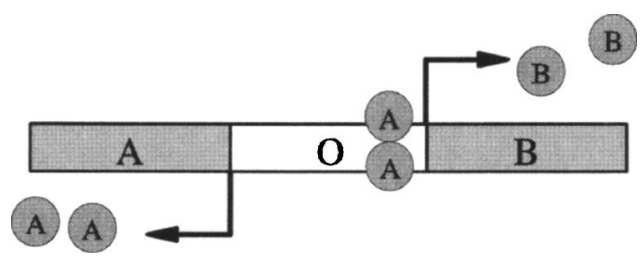

FIG. 8. Schematic representation of our model switch, corresponding to Eq. (28). Two divergently transcribed genes are under the control of a shared regulatory binding site on the DNA (the operator). Each protein can bind, in homodimer form, to the operator and block the production of the other species. either dimer), both genes can randomly be activated and produce either protein $\mathrm{A}$ or $\mathrm{B}$ with the same production rate $k_{\text {prod }}$, as in Eq. (28c). When an $\mathrm{A}_{2}$ dimer is bound to $\mathrm{O}$ [Eq. (28b)], the production of B is blocked. Conversely, when a $\mathrm{B}_{2}$ dimer is bound to $\mathrm{O}$ [Eq. (28b)], the production of $\mathrm{A}$ is blocked. Both proteins can decay in the monomer form (accounting for active degradation processes and dilution in a growing cell), as in Eq. (28e). We assume that transcription, translation, and protein folding can be modeled as a single Poisson process, representing protein production. Clearly, when one species is abundant over the other one, many dimers of the majority species will be created, and the probability of finding one of them bound to $\mathrm{O}$ will be high. This effect will in turn lower the production rate of the minority species, leading to a stabilization of the state. If a rare fluctuation, however, is able to build up a substantial number of the minority species, these will in turn dimerize and bind to $\mathrm{O}$, leading to a stochastic flip of the switch.

A mean field analysis carried out in $^{22}$ confirms this intuitive fact analytically: for suitable choices of the reaction rates, the system exhibits three fixed points: two symmetrical stable states, one rich in A and another rich in B, separated by one unstable state where the total number of $A$ equals the total number of B. The system can then be considered as a true bistable switch.

We have chosen parameters such that the system is bistable and symmetric. Using the production rate $k_{\text {prod }}^{-1}$ as a time unit, and indicating by $V$ the dimensionless volume of the system, we use $k_{f}=5 k_{\text {prod }} V, k_{b}=5 k_{\text {prod }}$ (so that the equilibrium dissociation constant for dimerization is $K_{D}^{d}=k_{d} / k_{f}$ $=1 / \mathrm{V}), k_{\mathrm{on}}=5 k_{\text {prod }}, k_{\text {off }}=k_{\text {prod }}$ [so that the equilibrium dissociation constant for operator binding is $K_{D}^{b}=k_{\text {off }} / k_{\text {on }}$ $=1 /(5 \mathrm{~V})], \mu=0.3 \mathrm{k}$. For simplicity, we will assume $V=1$. The system is simulated with an event-driven kinetic Monte Carlo algorithm ${ }^{32}$ which propagates the system according to the chemical master equation, thus accounting for the stochasticity arising from molecular discreteness and from the intrinsic randomness of reaction events. The simulation variables are the numbers of molecules $n$ (copy numbers) of each chemical species. Briefly, in this algorithm, one selects at each simulation step a waiting time until the next reaction, and an identity for the next reaction, from the correct probability distributions. One then advances the simulation time by the chosen waiting time, executes the chosen reaction, and updates the copy numbers of the species involved in the reaction.

A natural "order parameter" for the system is the difference between the total numbers of A and B proteins: $q=\lambda$ $=n_{\mathrm{A}}+2 n_{\mathrm{A}_{2}}+2 n_{\mathrm{OA}_{2}}-\left(n_{\mathrm{B}}+2 n_{\mathrm{B}_{2}}+2 n_{\mathrm{OB}_{2}}\right)$. Since the system is symmetric, we know that $\Phi_{A}=\Phi_{B}, k_{A B}=k_{B A}$, and therefore $p_{A}=p_{B}=0.5$. As this system is out of equilibrium, we do not sample a free-energy profile, but rather the nonequilibrium stationary probability distribution $\rho(q)=\rho(\lambda)$.

To measure the switching rate and $\rho(\lambda)$, we run a FFS simulation with 12 interfaces, setting $\lambda_{0}=-27, \lambda_{n}=27$, and using $N_{1}=10000$ points at the first interface. The interfaces are positioned as shown in Table V. We repeat the FFS sampling ten times to obtain error bars. The result is $k_{A B}=k_{B A}$ $=(8.66 \pm 0.07) \times 10^{-6} k_{\text {prod }}^{-1}$. From the FFS calculations, we 
TABLE V. Interfaces and the number of trials per interface for the FFS simulations for the model genetic switch.

\begin{tabular}{cccccc}
\hline \hline$i$ & $\lambda_{i}$ & $M_{i}$ & $i$ & $\lambda_{i}$ & $M_{i}$ \\
\hline 0 & -27 & 50000 & 6 & -8 & 250000 \\
1 & -25 & 50000 & 7 & -5 & 500000 \\
2 & -22 & 50000 & 8 & -2 & 500000 \\
3 & -18 & 50000 & 9 & 0 & 250000 \\
4 & -14 & 100000 & 10 & 10 & 50000 \\
5 & -12 & 100000 & 11 & 20 & 50000 \\
\hline \hline
\end{tabular}

also obtain the function $\psi_{A}(\lambda)=\psi_{A}(q)$ as described in Sec. VII, and since the system is symmetric, we can obtain $\psi_{B}(\lambda)$ from $\psi_{A}(\lambda)$ by a simple inversion transformation. Combining $\psi_{A}(\lambda)$ and $\psi_{B}(\lambda)$, we arrive at $\rho(\lambda)=\rho(q)$ for $-27<q<27$, which is plotted in Fig. 9 (a scaling factor is applied to account for the different normalization to the brute-force results). The distribution is clearly bimodal and shows symmetric peaks whose positions correspond to the stable solutions of the mean field equations (Ref. 22). As expected, a minimum in $\rho(\lambda)$ is observed for $\lambda=0$ (unstable solution of the mean field equations).

This system has a switching rate which is not exceedingly low, and we are also able to compute $\rho(q)$ using a brute force simulation of length $2 \times 10^{9} \mathrm{k}_{\text {prod. }}^{-1}$. The resulting stationary probability distribution is also shown in Fig. 9. Excellent agreement is obtained between the results of the FFS and brute-force calculations. Because the system spends little time in the region between the two basins, this part of $\rho(\lambda)$ is hard to calculate accurately with the brute-force run. The inset in Fig. 9 magnifies this region, showing the smooth profile produced by the FFS sampling.

\section{DISCUSSION}

The key concept used here to obtain the stationary distribution in the unstable region between two stable states $A$ and $B$ is to add the contributions from the trajectories that start in $A$ and go to $B$ or return to $A$, and those that start in $B$ and go to $A$ or return to $B$ (see Fig. 1). These contributions

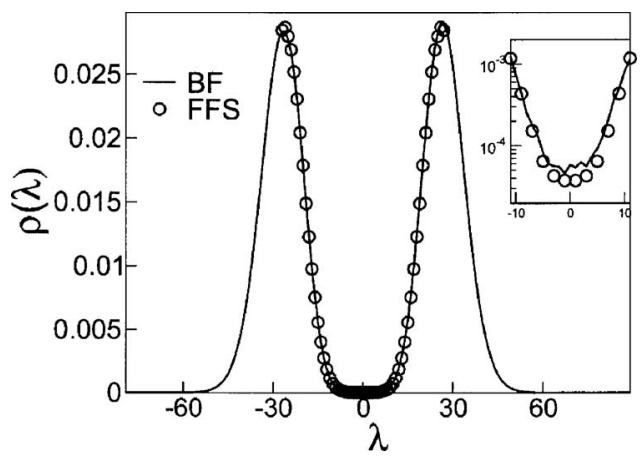

FIG. 9. Probability distribution as a function of the order parameter $\lambda$. The results are obtained both via long, steady state simulations (continuous line) and forward flux sampling (circles). The region around $|\lambda|=0$ can be accurately sampled only with FFS: the inset shows, on a logarithmic scale, a much smoother profile of the region close to the unstable steady state when FFS is used over brute force $(\mathrm{BF})$. A scaling factor has been applied to the FFS results since they were originally normalized over $-27<\lambda<27$ while the $\mathrm{BF}$ results are normalized over $-\infty<\lambda<\infty$. can be obtained by performing one FFS calculation starting in state $A$ and another starting in state $B$. For many rare event problems this is entirely possible; however, for systems where one state is very much more stable than the other, sampling the reverse transition $(B \rightarrow A)$ may be computationally difficult, even with FFS. We have encountered this problem in the Ising nucleation example discussed here in Sec. VIII. For equilibrium systems, this problem can be overcome by imposing an artificial stable state, as demonstrated here for the case of nucleation. However, this trick is not applicable for nonequilibrium systems. In general, in equilibrium systems the flux between any two state points is zero in steady state, while for nonequilibrium systems this need not be the case. In these nonequilibrium systems, the stationary distribution depends upon the full history of the trajectories. This, in general, prohibits the introduction of artificial boundaries. In particular, while for equilibrium systems detailed balance and microscopic reversibility dictate that the forward and backward transition paths have to occupy the same region in state-space, for systems that are out of equilibrium the backward and forward trajectories do not have to coincide; indeed, in these systems cycles in state space can occur. We have recently demonstrated that the switching pathways of genetic switches can follow such a scenario. ${ }^{11}$ If the forward and backward transition paths form a cycle in state space, then it is conceivable that the artificial stable state "short cuts" the cycle and generates a wrong ensemble of points from which trajectories are initiated in the reverse direction. It may be possible to devise alternative techniques for sampling the reverse transition in nonequilibrium systems, and this will be the subject of future work.

For the computation of free-energy barriers in equilibrium systems a wide range of numerical techniques is available. ${ }^{33}$ The advantage of the scheme proposed here is that the free energy can be directly obtained from a FFS simulation, obtaining simultaneously the rate constant, transition paths, and free energy landscape. This is important because both the calculation of rate constants and the evaluation of free-energy barriers are computationally demanding, especially for large and complex systems.

It has long been appreciated that free-energy barriers are critical quantities for understanding rare events in equilibrium systems, such as nucleation and protein folding. However, the "barriers," or minima in the stationary probabilities, that separate steady-states in nonequilibrium systems are equally important, because the rate of switching from one steady-state to the next is proportional to the probability of being at the top of the barrier. ${ }^{22}$ Some such barriers have recently been determined experimentally, including bimodal distributions of protein concentrations for genetic switches such as the one discussed in the previous section. ${ }^{34,35}$ To our knowledge, this technique is the first to be proposed for efficient computation of stationary distributions for rare events in multidimensional nonequilibrium systems. This should prove useful for enhancing our understanding of a range of important nonequilibrium rare event processes, as well as improving the efficiency of computation of free energy landscapes in equilibrium systems. 


\section{ACKNOWLEDGMENTS}

The authors thank Vitaly Shneidman, Beate Schmittmann, and Sorin Tănase-Nicola for their valuable advice. Part of this work falls under the research program of the "Stichting voor Fundamenteel Onderzoek der Materie (FOM)," which is financially supported by the "Nederlandse Organisatie voor Wetenschappelijk Onderzoek (NWO)." One of the authors (R.J.A.) was funded by the European Union Marie Curie program and by the Royal Society of Edinburgh.

\section{APPENDIX: COMPUTATION OF $\tau_{+}\left(q, \lambda_{0}\right)$}

In this appendix, we justify Eq. (6). Let us first imagine a very long brute-force simulation trajectory which meanders around the basin of attraction of $A$, making occasional excursions towards $B$. We can divide each of these excursion into portions separated by successive crossings of interfaces $\lambda_{0} \ldots \lambda_{n}$. Consider the portion of an excursion between its leaving $A$ and either reaching $\lambda_{1}$ or returning to $A$. We denote the distribution function (averaged over many excursions) for points visited during this portion $\nu_{0}(q)$. Likewise, the distribution function (averaged over many excursions) for points visited after crossing $\lambda_{1}$ and before reaching either $\lambda_{2}$ or $\lambda_{0}$ is denoted $\nu_{1}(q)$, and we can also obtain distribution functions $\nu_{i}(q)$ for all interfaces $0 \leqslant i<n$. It is important to note that the $\nu_{i}(q)$ are not normalized. In fact, the integral $\int d q \nu_{i}(q)$ contains information on the probability of an excursion reaching $\lambda_{i}$. Since our entire ensemble of excursions can be divided up in this way, we can write the entire distribution function $\tau_{+}\left(q ; \lambda_{0}\right)$ as the sum of contributions from all the portions of trajectories,

$$
\tau_{+}\left(q ; \lambda_{0}\right)=\sum_{i=0}^{n-1} \nu_{i}(q)
$$

Now let us consider the FFS procedure. Let us imagine we have generated a collection of points at interface $\lambda_{i}$. We fire $M_{i}$ trial runs from this collection of points and continue each one until either $\lambda_{0}$ or $\lambda_{i+1}$ is reached. We plot a histogram $\pi_{+}\left(q ; \lambda_{i}\right)$ of $q$ values for the points in this ensemble of trial runs. We have proved before ${ }^{12}$ that the distribution of these trial paths is identical to the distribution of corresponding portions of the "excursions" from $A$ in a brute-force simulation, except that it is reweighed by a factor that depends on the probability of reaching $\lambda_{i}$ from $A$, so that

$$
\pi_{+}\left(q ; \lambda_{i}\right)=\frac{\nu_{i}(q)}{P\left(\lambda_{i} \mid \lambda_{0}\right)}
$$

We have also proved before ${ }^{12}$ that

$$
P\left(\lambda_{i} \mid \lambda_{0}\right)=\prod_{j=0}^{i-1} P\left(\lambda_{j+1} \mid \lambda_{j}\right)
$$

for $i>0$ [for $\left.i=0, P\left(\lambda_{0} \mid \lambda_{0}\right)=1\right]$.

Combining (A2) and (A3) we arrive at

$$
\pi_{+}\left(q ; \lambda_{i}\right)=\frac{\nu_{i}(q)}{\prod_{j=0}^{i-1} P\left(\lambda_{j+1} \mid \lambda_{j}\right)}
$$

for $i>0$ and $\pi_{+}\left(q ; \lambda_{0}\right)=\nu_{0}(q)$. Rearranging Eq. (A4) and summing over interfaces, we arrive at

$$
\begin{aligned}
\tau_{+}\left(q ; \lambda_{0}\right) & =\sum_{i=0}^{n-1} \nu_{i}(q) \\
& =\pi_{+}\left(q ; \lambda_{0}\right)+\sum_{i=1}^{n-1} \pi_{+}\left(q ; \lambda_{i}\right) \prod_{j=0}^{i-1} P\left(\lambda_{j+1} \mid \lambda_{j}\right)
\end{aligned}
$$

which corresponds to Eq. (6).

${ }^{1}$ G. M. Torrie and J. P. Valleau, Chem. Phys. Lett. 28, 578 (1974).

${ }^{2}$ J. S. van Duijneveld and D. Frenkel, J. Chem. Phys. 96, 4655 (1992).

${ }^{3}$ P. R. ten Wolde, M. J. Ruiz-Montero, and D. Frenkel, J. Chem. Phys. 104, 9932 (1996).

${ }^{4}$ P. R. ten Wolde, M. J. Ruiz-Montero, and D. Frenkel, Faraday Discuss. 104, 93 (1996).

${ }^{5}$ D. Chandler, J. Chem. Phys. 68, 2959 (1978).

${ }^{6}$ C. H. Bennet, in Algorithms for Chemical Computations, ACS Symposium, Series No. 46, edited by R. Christofferson (American Chemical Society, Washington, D.C., 1977).

${ }^{7}$ C. Dellago, P. G. Bolhuis, F. S. Csajka, and D. Chandler, J. Chem. Phys. 108, 1964 (1998).

${ }^{8}$ C. Dellago, P. G. Bolhuis, and P. L. Geissler, Adv. Chem. Phys. 123, 1 (2002).

${ }^{9}$ T. S. van Erp, D. Moroni, and P. G. Bolhuis, J. Chem. Phys. 118, 7762 (2003).

${ }^{10}$ T. S. van Erp and P. G. Bolhuis, J. Comput. Phys. 205, 157 (2005).

${ }^{11}$ R. J. Allen, P. B. Warren, and P. R. ten Wolde, Phys. Rev. Lett. 94, 018104 (2005).

${ }^{12}$ R. J. Allen, D. Frenkel, and P. R. ten Wolde, J. Chem. Phys. 124, 024102 (2006).

${ }^{13}$ R. J. Allen, D. Frenkel, and P. R. ten Wolde, J. Chem. Phys. 124, 194111 (2006).

${ }^{14}$ L. R. Pratt, J. Chem. Phys. 85, 5045 (1986).

${ }^{15}$ P. G. Bolhuis, D. Chandler, C. Dellago, and P. L. Geissler, Annu. Rev. Phys. Chem. 53, 291 (2002).

${ }^{16}$ D. Moroni, P. G. Bolhuis, and T. S. van Erp, J. Chem. Phys. 120, 4055 (2004).

${ }^{17}$ D. Moroni, Ph.D. thesis, Universiteit van Amsterdam (2005).

${ }^{18}$ D. Moroni, T. S. van Erp, and P. G. Bolhuis, Phys. Rev. E 71, 056709 (2005).

${ }^{19}$ A. K. Faradjian and R. Elber, J. Chem. Phys. 120, 10880 (2004).

${ }^{20}$ W. E., W. Ren, and E. Vanden-Eijnden, Phys. Rev. B 66, 052301 (2002).

${ }^{21}$ L. Maragliano, A. Fischer, and E. Vanden-Eijnden, J. Chem. Phys. 125, 024106 (2006).

${ }^{22}$ P. B. Warren and P. R. ten Wolde, J. Phys. Chem. B 109, 6812 (2005).

${ }^{23}$ M. P. Allen and D. J. Tildesley, Computer Simulation of Liquids (Clarendon Press, Oxford, 1987).

${ }^{24}$ R. S. Maier and D. L. Stein, Phys. Rev. Lett. 69, 3691 (1992).

${ }^{25}$ R. S. Maier and D. L. Stein, J. Stat. Phys. 83, 291 (1996).

${ }^{26}$ R. S. Maier and D. L. Stein, Phys. Rev. E 48, 931 (1993).

${ }^{27}$ R. J. Allen, C. Valeriani, S. Tănase-Nicola, P. R. ten Wolde, and D. Frenkel (in preparation).

${ }^{28} \mathrm{~K}$. Binder, in Ising Model, edited by Springer Link Encyclopaedia of Mathematics (Springer, Berlin, 2001).

${ }^{29}$ R. P. Sear, J. Phys. Chem. B 110, 4985 (2006).

${ }^{30}$ K. F. Kelton, in Crystal Nucleation in Liquids and Glasses, Vol. 45, edited by H. Ehrenreich and D. Turnbull (Academic Press, Boston, 1991).

${ }^{31}$ L. Onsager, Phys. Rev. 65, 117 (1944).

${ }^{32}$ D. T. Gillespie, J. Phys. Chem. 81, 2340 (1977).

${ }^{33}$ D. Frenkel and B. Smit, Understanding Molecular Simulation. From Algorithms to Applications (Academic Press, Boston, 2002), 2nd ed.

${ }^{34}$ T. S. Gardner, C. R. Cantor, and J. J. Collins, Nature 403, 339 (2000).

${ }^{35}$ E. M. Ozbudak, M. Thattai, I. Kurtser, A. D. Grossman, and A. van Oudenaarden, Nat. Genet. 31, 69 (2002). 